\title{
Variations in the Profiles of Vascular- Related Factors Among Different Sub-Types of Polycystic Ovarian Syndrome in Northern China
}

\author{
Mei-mei Liu ${ }^{*}$ Xiu-hui Chen, Xiu-min Lu, Fang-fang Wang, Chao Wang, Yu Liu, \\ Pei-ling Li, Bo-tao Du, Sha Liang, Pi-dong Gong and Yu-xin Wang \\ Department of Gynaecology and Obstetrics, The Second Affiliated Hospital of Harbin Medical University, Harbin, China
}

OPEN ACCESS

Edited by:

Damian G. Romero,

University of Mississippi Medical

Center, United States

Reviewed by:

Qi Yu,

Peking Union Medical College

Hospital (CAMS), China

Carolina Dalmasso,

University of Kentucky, United States

${ }^{*}$ Correspondence:

Mei-mei Liu

liumm197723@126.com

Specialty section:

This article was submitted to

Reproduction,

a section of the journal

Frontiers in Endocrinology

Received: 02 April 2020 Accepted: 04 December 2020

Published: 26 February 2021

Citation:

Liu M-m, Chen X-h, LuX-m, Wang F-f, Wang C, Liu Y, Li P-I, Du B-t, Liang S,

Gong $P-d$ and Wang Y-x (2021) Variations in the Profiles of VascularRelated Factors Among Different Sub-

Types of Polycystic Ovarian Syndrome in Northern China.

Front. Endocrinol. 11:527592. doi: 10.3389/fendo.2020.527592
Recently, a growing body of evidence has suggested that abnormal ovarian angiogenesis, secondary to the imbalance between various angiogenic markers, is involved in the pathogenesis of PCOS, and this has led to the use of various interventions (such as Diane35) to restore the normal ovarian angiogenesis. Therefore, we conducted the current investigation to determine the role of such markers (endothelial growth factor (VEGF), endostatin (ES), and thrombospondin-1 (TSP-1)) in the pathogenesis of PCOS along with the associated changes in ovarian blood flow in patients with PCOS compared to healthy controls, both before and after a course of oral contraception. A total of 381 patients with PCOS and 98 healthy females of childbearing age were recruited from July 2014 to June 2017 at the Reproductive Center of the Second Affiliated Hospital of Harbin Medical University. The serum levels of VEGF, ES, and TSP-1 were determined by enzyme-linked immunosorbent assay, while ovarian perfusion was measured by the pulsatility index (PI) and resistance index (RI) by using transvaginal color Doppler ultrasound. Repeated analyses were carried out after 3 months of Diane-35 treatment. Post-treatment serum levels of luteinizing hormone $(\mathrm{LH})$ /follicle stimulating hormone $(\mathrm{FSH})$ ratio of patients with PCOS decreased significantly $(\mathrm{P}<0.05)$. The $\mathrm{RI}$ values of most $\mathrm{PCOS}$ patients increased after treatment $(P<0.05)$, while $P$ I was significantly increased in all patients $(P<0.05)$. However, variable changes in the serum levels of TSP-1, VEGF, and ES after treatment were observed. Serum VEGF levels showed a negative correlation with serum LH/FSH ratio, $T$ concentration, and ES $(P<0.05)$, while ES levels were negatively correlated with serum $T$ concentrations only $(P<0.05)$. The markers of angiogenesis (VEGF, ES, and TSP1) were expressed differently among PCOS patients, who also responded differently to the same course of Diane-35 treatment. This field still warrants further investigation to reach a more definitive conclusion.

Keywords: polycystic ovarian syndrome, vascular-related factors, vascular endothelial growth factor, endostatin, thrombospondin-1 


\section{INTRODUCTION}

Polycystic ovary syndrome (PCOS), a gynecological and endocrinal disorder in women, has been recently characterized by the disruption of ovarian blood flow and angiogenesis (1). The prevalence of PCOS is 5\%-7\% among women of childbearing age (19-25 years). Despite being variable in different populations, the main presentations of PCOS include ovulation disorders (oligo or amenorrhea), hyperandrogenism (HA), insulin resistance (IR), and metabolic or psychological disturbances $(2,3)$. To date, the pathogenesis of PCOS has not been well-studied. However, it has been proposed that ovarian angiogenesis plays a crucial role in the pathogenesis of PCOS, given the fact that the formation and regression of blood vessels are mandatory for proper follicle maturation, ovulation, and corpus luteum formation (1). Therefore, it was proposed that such vascular alterations, controlled by various angiogenic markers, would be responsible for the ovarian features of PCOS (4).

Vascular endothelial growth factor (VEGF), which is responsible for promoting the proliferation of vascular endothelial cells and regulating vascular permeability, is of great importance in follicular development (5). Endostatin (ES), an anti-angiogenic factor, interferes with the proangiogenic action of VEGF and inhibits the proliferation of endothelial cells (ECs) while suppressing the formation of new blood vessels (6). Thrombin sensitive protein-1 (TSP-1), known as thrombospondin-1, is also an important inhibitor of angiogenesis, acting by inducing apoptosis in ECs by targeting VEGF (6).

A growing body of evidence suggests that VEGF, ES, and TSP-1 are abnormally expressed in patients with PCOS $(7,8)$.

Moreover, it is proposed that the disruption of normal ovarian angiogenesis, secondary to the imbalance between angiogenic and anti-angiogenic factors, is responsible for the occurrence of several pathologies in PCOS, including abnormal follicle development, increased quantity of small follicles, anovulation, and cyst formation (1). Consequently, various interventions (such as oral contraceptive pills and metformin) have been proposed as therapeutic options for PCOS, acting by restoring the normal ovarian angiogenesis $(9,10)$.

Therefore, we conducted this study to determine the role of alterations in the expression of the aforementioned angiogenic markers in the pathogenesis of PCOS, along with the associated changes in ovarian blood flow. We also investigated the changes in the expression of these markers following the use of Diane-35 in women with PCOS.

\section{MATERIALS AND METHODS}

\section{Subjects}

In this prospective case-control study recruited 381 patients with PCOS who attended the Reproductive Center of the Second Affiliated Hospital of Harbin Medical University from July 2014 to June 2017. In addition, we included 98 healthy women of childbearing age, who received intracytoplasmic sperm injection due to male factor infertility as the control group. This research is approved by the Ethics Committee of the Second Affiliated Hospital of Harbin Medical University (KY2018-043).

\section{Inclusion Criteria}

We followed the recommendations of the Rotterdam International Conference (11) and the Chinese PCOS diagnostic criteria (2011) (12) in diagnosing women with PCOS. The diagnosis of PCOS was reached if 2 or 3 items of the following criteria were fulfilled:

(1) Clinical manifestations of oligo and/or anovulation, which included:

a. Oligomenorrhea: the menstrual cycle is from 35 days to 6 months per year.

b. Amenorrhea: either secondary amenorrhea (menopause period $\geq 6$ months) or primary amenorrhea (no menarche at 16 years old).

c. Irregular uterine bleeding: irregular menstrual cycle or period or menstrual flow.

(2) Clinical and/or biochemical signs of hyperandrogenism, which included:

a. Symptoms of HA: acne, hypersexual hair, obesity, black acanthosis, etc.

b. Biochemical determination of serum reproductive hormone concentration: biochemical HA

The laboratory diagnosis of HA in our center was determined if the total serum testosterone level was $>0.48 \mathrm{ng} / \mathrm{ml}$.

(3) Ultrasound examination showing polycystic ovarian changes:

a. $\geq 12$ follicles with a diameter of $2-9 \mathrm{~mm}$ in one or both ovaries, and/or:

b. ovarian volume $\geq 10 \mathrm{~cm} 3$ [ovary volume is $0.5 \times$ longitudinal diameter $(\mathrm{cm}) \times$ transverse diameter $(\mathrm{cm}) \times$ forward and backward diameter $(\mathrm{cm})]$.

Patients were included when they fulfilled at least two of the mentioned criteria, with the exclusion of other causes at the same time. Moreover, patients with other causes of hyperandrogenism were also excluded, such as abnormal thyroid function, hyperprolactinemia, delayed-type adrenal hyperplasia, 21hydroxylase deficiency, Cushing syndrome, schizophrenia syndrome, primary hypoovarian dysfunction, or premature ovarian failure, ovarian or adrenal androgen-producing tumors, and functional hypothalamic amenorrhea.

Patients were then stratified according to the presenting manifestations into four groups of PCOS phenotypes: group A (patients who fulfilled criteria 1,2, and 3), group B (patients who fulfilled criteria 1 and 3), group C (patients who fulfilled criteria 1 and 2), and group D (patients who fulfilled criteria 2 and 3 ).

On the other hand, the inclusion criteria of the normal control group were as follows: (1) healthy women of childbearing age, who are unable to bear children due to male factor infertility, (2) women with normal menstruation and without any clinical manifestations of PCOS, (3) women with no endocrinal disorders, such as abnormal sex hormone levels, abnormal oral glucose tolerance test (OGTT), or insulin resistance (IR), and (4) women with no 
evidence of abnormal changes in the uterus or the ovaries based on ultrasound examination.

\section{Exclusion Criteria}

Individuals in the control group were excluded if they had systemic inflammatory diseases, thyroid disease, hyperprolactinemia, adrenal disease, hypertension, diabetes, or other complications. Those who had used hormonal drugs in the past 6 months prior to participation in the study were also excluded.

\section{Collection of Blood Samples}

All study participants were enrolled at the early follicular stage (days 2-4 of the menstrual cycle). If no dominant follicles were found in both ovaries by transvaginal ultrasound examination, a $5 \mathrm{ml}$ sample of peripheral venous blood was collected from fasting patients in the morning and placed in a polypropylene microcentrifuge tube. The tube contents were centrifuged at $2,000 \mathrm{r} / \mathrm{min}$, and the serum was then frozen and stored in a refrigerator at $-20^{\circ} \mathrm{C}$ for later use.

\section{Determination of Sex Hormone Levels}

Peripheral venipuncture, during the early follicular phase of the menstrual cycle (days 2-4), was done to collect blood samples for hormonal level estimation following overnight fasting. Electrochemiluminescence immunoassay was used to measure the levels of sex hormones, including luteinizing hormone (LH), follicle-stimulating hormone (FSH), and total testosterone (T). The corresponding sex hormone kits were purchased from Tosoh Corporation, Japan. The remaining serum was stored in a refrigerator at $-20^{\circ} \mathrm{C}$ for later use.

\section{Determination of Serum VEGF, ES, and TSP-1 Levels}

The primary outcome of our study was to determine the changes in the expression of angiogenic markers (VEGF, ES, and TSP-1) along with the associated alterations in ovarian blood flow) in women with PCOS compared to healthy controls before and after a course of Diane-35 treatment.

The serum levels of VEGF, ES, and TSP-1 were measured from the remaining patients' serum by immunosorbent assay (ELISA) following the manufacturer's manual (AIA-2000 automatic immunoluminescence analyzer Japan Tosoh Co. Ltd). ELISA kits were purchased from (Shanghai Baili Biotechnology Co. Ltd.) and (Nanjing Heikeer Biotechnology Co. Ltd, Japan).

\section{Measurement of Ovarian Interstitial Blood Flow}

On the day of blood collection, transvaginal color Doppler ultrasound was used to monitor ovarian interstitial blood flow (ProSound $\alpha 7$ Doppler color ultrasound Aloca, Japan). Pulsatility (PI) and resistance indices (RI) were used to measure ovarian blood flow. This process was conducted by the same clinician. In each case, the volume of ovarian interstitial blood flow on each side was taken three times, and the average value was then calculated and analyzed.

\section{Compound Cyproterone (Diane-35) Treatment}

Patients with PCOS received oral Diane-35 on the 5th day of their menstrual periods. Diane-35 is made up of Ethinylestradiol $(35 \mu \mathrm{g})$ and Cyproterone acetate $(2 \mathrm{mg})$, and patients were given this drug at a dose of one tablet a day for 21 days per cycle. They continued receiving this medication for three successive cycles. After that, the serum levels of the aforementioned markers were reassessed.

\section{Statistical Analysis}

We used the Statistical Package for Social Sciences (SPSS) software (Version 23.0) to conduct our analyses. Data were expressed as means \pm standard deviations $(\bar{\chi} \pm \mathrm{SD})$. Normality (Shapiro-Wilk) and homogeneity (Levene) tests were conducted to test the distribution of the data. If the data were normally and homogeneously distributed, the differences among multiple groups were analyzed through a one-way analysis of variance (ANOVA). Post-hoc comparisons of each group were performed using the LSD test, while the Paired t-test was used to draw comparisons between groups before and after treatment. The two-tailed Pearson test was used to establish correlations between analyzed variables. A value of $\mathrm{P}<0.05$ was considered the cut-off value for statistical significance.

\section{RESULTS}

\section{Demographic and Clinical Characteristics}

A total of 381 patients with PCOS were included in the case group, with 94, 103, 95, and 89 patients in groups A, B, C, and D, respectively. The mean age in each group did not differ significantly from the control group $(\mathrm{P}=0.337)$. However, baseline BMI was significantly higher in all PCOS groups compared to control $(\mathrm{P}<0.05)$. Serum $\mathrm{LH} / \mathrm{FSH}$ ratio and $\mathrm{T}$ levels in all groups of PCOS patients were significantly higher than those in the control group $(\mathrm{P}<0.05)$. Ovarian artery PI and RI values were significantly lower in patients with PCOS in all groups compared to the control group $(\mathrm{P}<0.05)$. Similarly, serum TSP-1 levels in all PCOS groups were significantly lower than the control group $(\mathrm{P}<0.05)$. On the other hand, serum ES levels in all PCOS groups were significantly higher than those recorded in the control group $(\mathrm{P}<0.05)$. Noteworthy, serum VEGF levels were significantly higher in group B only when compared to the control group (980.5 vs. $620.4, \mathrm{P}<0.001)$. On the other hand, serum VEGF levels in groups $\mathrm{A}, \mathrm{C}$, and D were lower compared to the control group; however, this difference did not reach statistical significance $(P>0.05)$ (Table 1). Figure 1 shows the ultrasonic images of ovarian blood flow and the reported PI value during the assessment before Diane-35 treatment in the control group and the various PCOS phenotypes (A, B, and D).

\section{Clinical and Biochemical Characteristics After Diane-35 Treatment}

Following the treatment with Diane-35, the serum levels of LH/ $\mathrm{FSH}$ ratio in all PCOS groups were significantly reduced 
TABLE 1 | Demographic characteristics and laboratory parameters of included patients in each group at baseline.

\begin{tabular}{|c|c|c|c|c|c|c|}
\hline \multirow[t]{2}{*}{ Variable } & \multicolumn{4}{|c|}{ PCOS groups } & \multirow[t]{2}{*}{ Control $(\mathrm{N}=98)$} & \multirow[t]{2}{*}{ P-value } \\
\hline & Group A (N=94) & Group B $(N=103)$ & Group C (N=95) & Group D (N=89) & & \\
\hline Age (years) & $25.4 \pm 3.5$ & $24.7 \pm 3.2$ & $25.1 \pm 3.2$ & $24.6 \pm 3.0$ & $25.4 \pm 3.9$ & 0.337 \\
\hline BMI (Kg/m2) & $26.2 \pm 4.1^{\star}$ & $25.2 \pm 4.7^{\star}$ & $25.2 \pm 4.3^{\star}$ & $25.2 \pm 4.5^{\star}$ & $22.1 \pm 3.8$ & 0.000 \\
\hline LH/FSH ratio & $2.5 \pm 1.0^{\star}$ & $2.0 \pm 0.9^{\star \#}$ & $2.9 \pm 1.3^{\star \# \&}$ & $2.8 \pm 1.4^{\star \&}$ & $1.5 \pm 1.0$ & 0.000 \\
\hline $\mathrm{T}$ (ng/ml) & $0.6 \pm 0.2^{*}$ & $0.3 \pm 0.1^{\#}$ & $0.5 \pm 0.1^{\star \# \&}$ & $0.5 \pm 0.1^{\star \# \&}$ & $0.3 \pm 0.1$ & 0.000 \\
\hline RI & $0.6 \pm 0.2^{*}$ & $0.5 \pm 0.2^{*}$ & $0.6 \pm 0.2^{\star \&}$ & $0.5 \pm 0.2^{\star \$}$ & $0.7 \pm 0.2$ & 0.000 \\
\hline PI & $1.7 \pm 0.8^{\star}$ & $1.4 \pm 0.4^{\star \#}$ & $1.5 \pm 0.4^{\star \#}$ & $1.5 \pm 0.4^{\star \#}$ & $2.1 \pm 0.8$ & 0.000 \\
\hline TSP-1 (ng/ml) & $161.0 \pm 24.5^{\star}$ & $166.4 \pm 24.6^{\star}$ & $179.3 \pm 23.6^{\star \# \&}$ & $169.5 \pm 15.8^{\star \# \$}$ & $189.4 \pm 23.1$ & 0.000 \\
\hline VEGF (pg/ml) & $606.5 \pm 132.3$ & $980.5 \pm 174.6^{\star \#}$ & $595.8 \pm 118.2 \&$ & $581.2 \pm 139.0^{\&}$ & $620.4 \pm 108.1$ & 0.000 \\
\hline ES (ng/ml) & $269.8 \pm 25.6^{\star}$ & $289.2 \pm 26.8^{\star \#}$ & $256.1 \pm 23.1^{\star \#} \&$ & $273.0 \pm 34.0^{\star \& \$}$ & $177.8 \pm 21.9$ & 0.000 \\
\hline HOMA-IR & $5.3 \pm 3.1^{\star}$ & $4.9 \pm 2.0^{\star}$ & $5.3 \pm 2.2^{\star}$ & $3.8 \pm 1.4^{\star \# \& \$}$ & $2.2 \pm 0.6$ & 0.000 \\
\hline
\end{tabular}

*significant difference compared to control; " significant difference compared to group A; ${ }^{\&}$ significant difference compared to group B; ${ }^{\$}$ significant difference compared to group D; BMI, Body Mass Index; LH, Luteinizing Hormone; FSH, Follicle Stimulating Hormone; RI, Resistance Index; PI, Pulsatility Index; TSP-1, Thrombospondin-1; VEGF, Vascular Endothelial Growth Factor; ES, Endostatin; HOMA-IR, Homeostasis Model Assessment of Insulin Resistance.

A

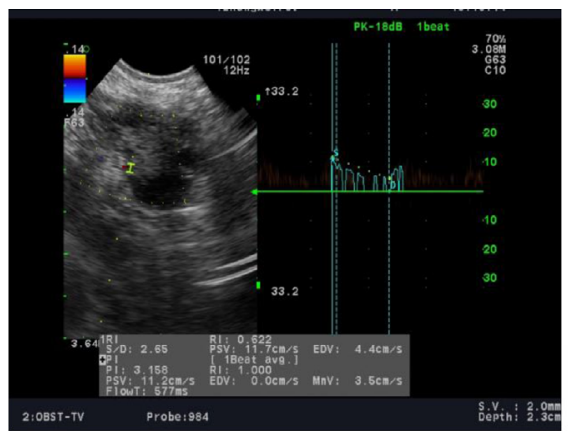

C

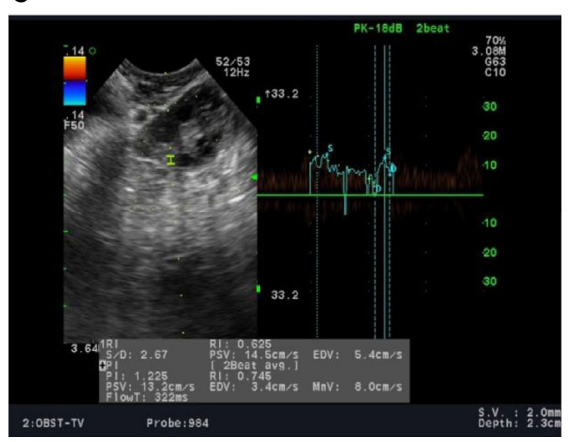

B

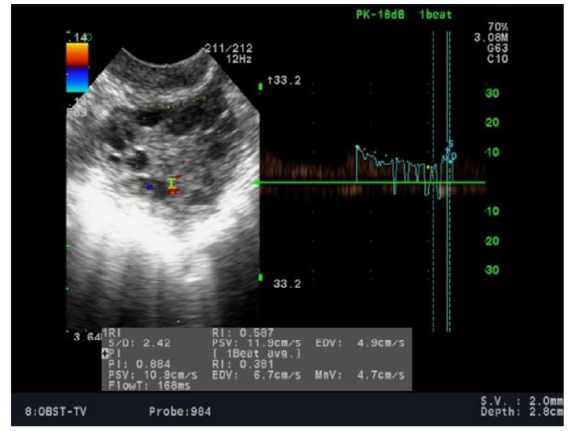

D

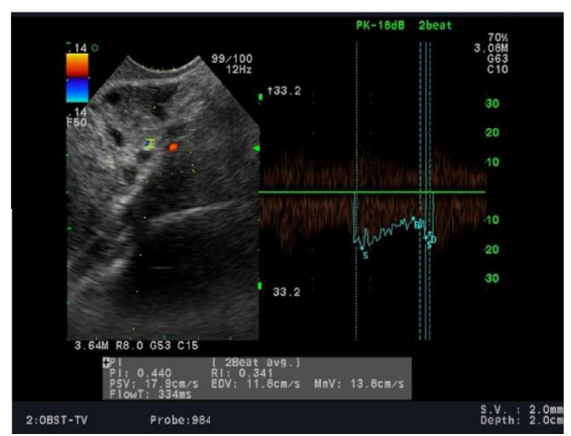

FIGURE 1 | Ultrasound recording of the ovarian blood flow in: (A) a normal female; (B-D) in patients with PCOS stratified into phenotypes A, B, and D, respectively, as described in the methods section.

compared to pre-treatment levels $(\mathrm{P}<0.05)$. Similarly, serum $\mathrm{T}$ concentration was significantly lower after treatment in all groups except for group B, which did not show statistical significance. After treatment, both PI and RI values were significantly increased in all PCOS groups compared to pretreatment levels; however, group $\mathrm{C}$ did not show any statistically significant difference regarding RI. In the same context, serum TSP-1 indices were significantly increased in groups A, B, and D after treatment $(\mathrm{P}<0.05)$. In contrast, serum VEGF levels were significantly reduced after treatment in groups $\mathrm{B}$ and $\mathrm{C}$ only $(\mathrm{P}<$
0.05). Moreover, serum ES levels in groups $\mathrm{A}, \mathrm{B}$, and $\mathrm{C}$ were significantly decreased after treatment $(P<0.05)$ (Table 2). Figure 2 shows the ultrasonic images of ovarian blood flow and the reported PI value after a course of Diane- 35 treatment in the various PCOS phenotypes (A-D).

\section{Correlations Between Biological Indicators and Ovarian Blood Flow}

Serum VEGF levels were found to be negatively correlated with serum LH/FSH ratio $(r=-0.180)$, $\mathrm{T}$ concentrations $(\mathrm{r}=$ 
TABLE 2 | Changes in ovarian functions among PCOS patients following a course of Diane-35 treatment.

\begin{tabular}{|c|c|c|c|c|c|c|c|c|c|c|c|c|}
\hline \multirow[t]{2}{*}{ Parameter } & \multicolumn{3}{|c|}{ Group A (N = 94) } & \multicolumn{3}{|c|}{ Group B (N = 103) } & \multicolumn{3}{|c|}{ Group C (N = 95) } & \multicolumn{3}{|c|}{ Group D (N = 89) } \\
\hline & $\begin{array}{c}\text { Before } \\
\text { treatment }\end{array}$ & $\begin{array}{c}\text { After } \\
\text { treatment }\end{array}$ & $\mathbf{P}$ & $\begin{array}{c}\text { Before } \\
\text { treatment }\end{array}$ & $\begin{array}{c}\text { After } \\
\text { treatment }\end{array}$ & $\mathbf{P}$ & $\begin{array}{c}\text { Before } \\
\text { treatment }\end{array}$ & $\begin{array}{c}\text { After } \\
\text { treatment }\end{array}$ & $\mathbf{P}$ & $\begin{array}{c}\text { Before } \\
\text { treatment }\end{array}$ & $\begin{array}{c}\text { After } \\
\text { treatment }\end{array}$ & $\mathbf{P}$ \\
\hline $\begin{array}{l}\text { LH/FSH } \\
\text { ratio }\end{array}$ & $2.51 \pm 0.96$ & $2.04 \pm 0.61$ & 0.000 & $2.03 \pm 0.91$ & $1.61 \pm 0.43$ & 0.000 & $2.87 \pm 1.30$ & $1.70 \pm 0.5$ & 0.000 & $2.75 \pm 1.43$ & $1.76 \pm 0.52$ & 0.000 \\
\hline T (ng/ml) & $0.59 \pm 0.17$ & $0.45 \pm 0.08$ & 0.000 & $0.28 \pm 0.09$ & $0.29 \pm 0.08$ & 0.479 & $0.51 \pm 0.09$ & $0.39 \pm 0.10$ & 0.000 & $0.50 \pm 0.09$ & $0.40 \pm 0.4$ & 0.000 \\
\hline RI & $0.57 \pm 0.24$ & $0.87 \pm 0.22$ & 0.000 & $0.52 \pm 0.17$ & $0.73 \pm 0.22$ & 0.000 & $0.59 \pm 0.17$ & $0.61 \pm 0.20$ & 0.544 & $0.53 \pm 0.19$ & $0.72 \pm 0.22$ & 0.000 \\
\hline PI & $1.72 \pm 0.76$ & $1.93 \pm 0.28$ & 0.012 & $1.36 \pm 0.41$ & $1.91 \pm 0.42$ & 0.000 & $1.48 \pm 0.43$ & $1.88 \pm 0.61$ & 0.000 & $1.51 \pm 0.39$ & $1.90 \pm 0.50$ & 0.000 \\
\hline $\begin{array}{l}\text { TSP-1 (ng/ } \\
\text { ml) }\end{array}$ & $161.0 \pm 24.5$ & $\begin{array}{c}180.62 \pm \\
20.93\end{array}$ & 0.000 & $166.4 \pm 24.6$ & $\begin{array}{c}174.13 \pm \\
20.45\end{array}$ & 0.022 & $\begin{array}{c}179.27 \pm \\
23.55\end{array}$ & $\begin{array}{c}182.56 \pm \\
22.79\end{array}$ & 0.297 & $\begin{array}{c}169.49 \pm \\
15.83\end{array}$ & $\begin{array}{c}175.72 \pm \\
21.91\end{array}$ & 0.021 \\
\hline $\begin{array}{l}\text { VEGF (pg/ } \\
\mathrm{ml})\end{array}$ & $\begin{array}{c}606.5 \pm \\
132.3\end{array}$ & $\begin{array}{c}589.48 \pm \\
123.47\end{array}$ & 0.368 & $\begin{array}{c}980.5 \pm \\
174.6\end{array}$ & $\begin{array}{c}896.34 \pm \\
120.72\end{array}$ & 0.000 & $\begin{array}{c}595.79 \pm \\
118.20\end{array}$ & $\begin{array}{c}553.95 \pm \\
132.05\end{array}$ & 0.038 & $\begin{array}{c}581.17 \pm \\
139.02\end{array}$ & $\begin{array}{c}550.79 \pm \\
100.54\end{array}$ & 0.074 \\
\hline ES (ng/ml) & $269.8 \pm 25.6$ & $\begin{array}{c}258.25 \pm \\
25.37\end{array}$ & 0.003 & $289.2 \pm 26.8$ & $\begin{array}{c}229.52 \pm \\
25.70\end{array}$ & 0.000 & $\begin{array}{c}256.07 \pm \\
23.09\end{array}$ & $\begin{array}{c}217.85 \pm \\
24.67\end{array}$ & 0.000 & $\begin{array}{c}273.98 \pm \\
34.03\end{array}$ & $\begin{array}{c}265.69 \pm \\
27.82\end{array}$ & 0.136 \\
\hline
\end{tabular}

BMI, body mass index; LH, luteinizing hormone; FSH, follicle stimulating hormone; RI, Resistance Index; PI, Pulsatility Index; TSP-1, thrombospondin-1; VEGF, vascular endothelial growth factor; ES, endostatin.

A

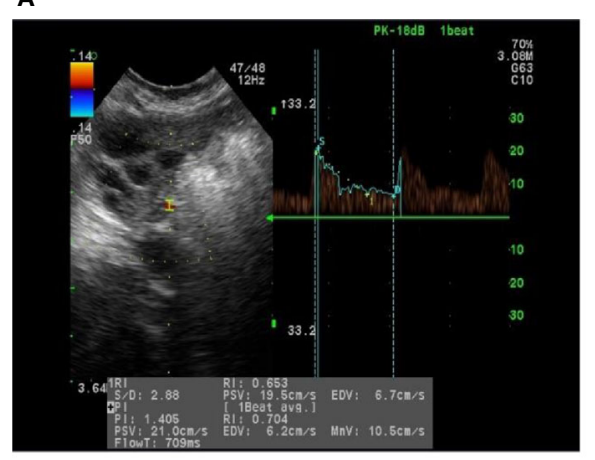

C

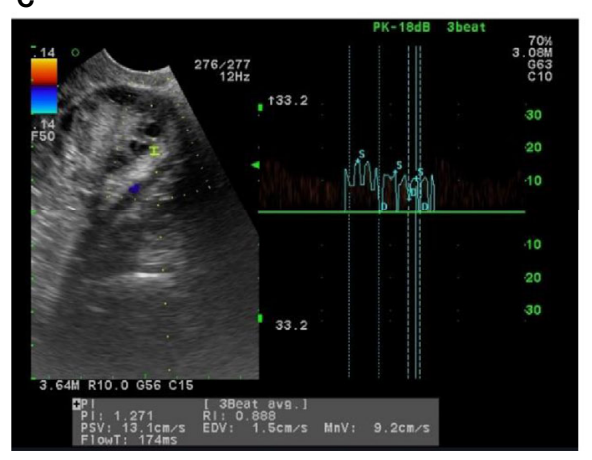

B

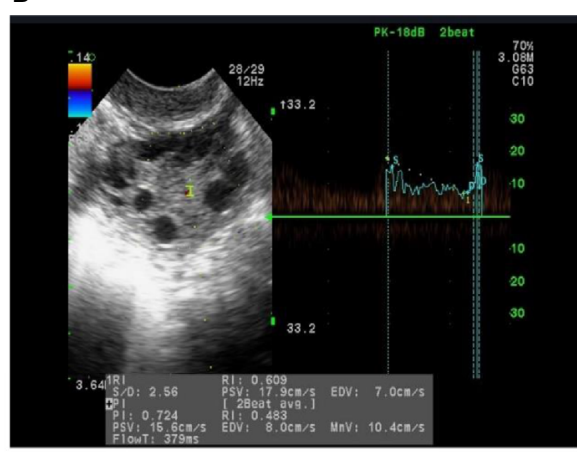

D

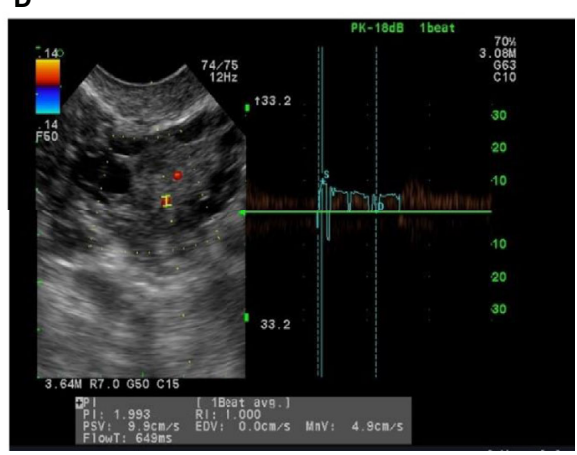

FIGURE 2 | Images of ultrasound recordings of ovarian blood flow among PCOS patients after Diane-35 treatment. Images (A-D) are of representative patients in groups (A-D), as described in the methods section.

-0.581), and PI $(\mathrm{r}=-0.138)(\mathrm{P}<0.05)$. However, we found that VEGF levels were positively correlated with ES levels $(r=$ $0.266, \mathrm{P}<0.05)$. On the other hand, ES levels were only negatively correlated with serum $\mathrm{T}$ concentrations $(\mathrm{r}=$ $-0.237, \mathrm{P}<0.05)$. There were no correlations between serum TSP-1 levels with any other biological indicators, PI, or RI (P $>0.05)$. None of the three biomarkers depicted a correlation with RI (Figure 3).

\section{DISCUSSION}

Ovarian blood flow perfusion reflects the phases of follicular development and the accompanying hormonal changes. This can be monitored by the occurrence of ovulation as well as the formation and degradation of the corpus luteum (13). The hemodynamic parameters RI and PI reflect the resistance of ovarian arterial blood flow, where lower values suggest a greater 
A

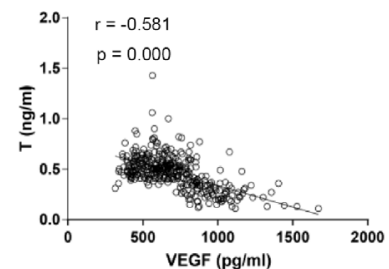

D

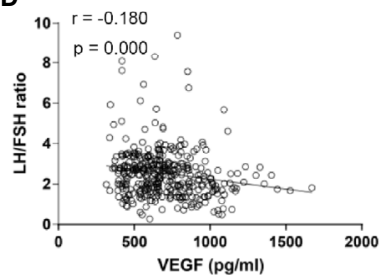

B

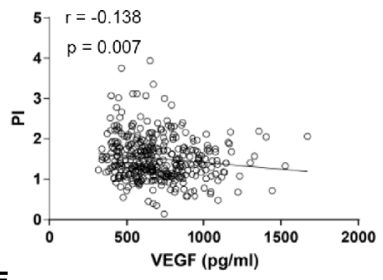

E

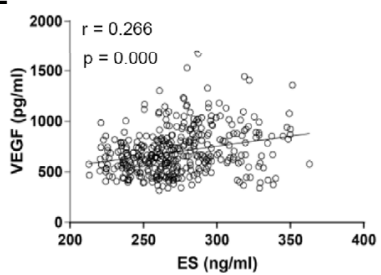

C

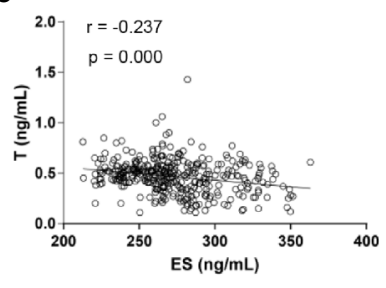

FIGURE 3 | Scatter plots for the correlation analysis of the expression of serum VEGF, ES and serum sex hormones among PCOS patients. (A) Correlation analysis between serum VEGF levels and serum T concentrations ( $r=-0.581, P<0.001)$; (B) Correlation analysis between serum VEGF levels and PI $(r=-0.138$, $P=0.007)$; (C) Correlation analysis between serum ES levels and serum T concentrations ( $r=-0.237, P<0.001)$; (D) Correlation analysis between serum VEGF levels and LH/ FSH ratio ( $r=-0.180, P<0.001)$; (E) Correlation analysis between serum ES levels and serum VEGF levels $(r=0.266, P<0.001)$. T, Testosterone; VEGF, Vascular Endothelial Growth Factor; ES, Endostatin; LH/FSH, Luteinizing Hormone/Follicular Stimulating Hormone; PI, Pulsatility Index; P, P-value.

degree of perfusion (14). In this study, we found that the ovarian interstitial blood flow in patients with PCOS was significantly increased (lower RI and PI values) compared to healthy controls. Going in line with other studies, the ultrasound results from our study revealed that ovarian interstitial blood flow, which was rich and with round and blunt peaks, was also characterized with high speed and low-resistance $(8,15,16)$.

It has been reported that changes in ovarian blood flow are associated with high serum LH and insulin levels in patients with PCOS (17). Additionally, it is speculated that the abnormal perfusion of ovarian blood flow observed among PCOS patients is closely related to endocrinal disorders, which may be implicated in the ovulation disorders displayed by patients with PCOS (18). Moreover, abnormalities in ovarian angiogenesis that were observed in many patients with PCOS are accompanied by changes in the expression of various cytokines, which are known to regulate angiogenesis (7).

For instance, VEGF is a highly effective angiogenic factor, promoting the proliferation of vascular endothelial cells and tube formation through the action of tyrosine kinase receptors (19). VEGF plays a role in maintaining the dynamic balance of blood flow around the follicles (19). It increases the capillary permeability with subsequent extravasation of plasma proteins, which results in the increase of the ovarian matrix. This, in turn, influences the development and maturation of follicles (20). It also acts by promoting endothelial proliferation, migration, and survival (21). Some studies reported that increased ovarian vascularity is associated with an increase in serum VEGF levels in patients with PCOS $(22,23)$. In our study, VEGF levels of PCOS subtypes were variable compared to the control group. All PCOS subtypes, except for subtype B, had lower mean VEGF values compared to the control group; however, these differences did not reach statistical significance, which may be attributable to the small sample size of each group. This goes in line with the literature (24). Of note, PCOS subtype B (normal androgen levels) had significantly higher mean VEGF levels compared to the control group as well as other PCOS subtypes. This is inconsistent with the current evidence that suggests that increased androgens activate hypoxia-inducible factor 1 (HIF1) $(25,26)$, a documented transcriptional activator of VEGF (27), and this androgen receptor-mediated effect is amplified in hypoxic conditions $(28,29)$. Various factors have been proposed to affect VEGF levels in women with PCOS. For example, vitamin D supplementation reduces VEGF levels in women with PCOS (24). This potential confounder was not studied in our study, and the heterogeneity between our findings and the available evidence should be thoroughly investigated in future research.

$\mathrm{ES}$, on the other hand, is a potent inhibitor of angiogenesis. The biological actions of ES include inhibition of angiogenesis of tumor cells and induction of apoptosis. As a result, ES inhibits the formation of new blood vessels; however, it has no effect on present healthy vessels (30). Studies have suggested that ES can specifically inhibit the proliferation of vascular endothelial cells and block the pro-angiogenic effect of VEGF (31). Moreover, ES can also inhibit endothelial cell nitric oxide synthase (eNOS) phosphorylation, induce eNOS inactivation, and reduce VEGFinduced NO synthesis. These effects lead to the inhibition of VEGF-mediated angiogenesis and vascular permeability (32). VEGF acts mainly by binding to endothelial cell receptors, mainly VEGFR-1 (vascular endothelial growth factor receptor 1), which is also known as soluble fms-like tyrosine kinase-1 (sFlt1). On the other hand, ES inhibits angiogenesis by directly binding to sFlt-1 and interfering with VEGF-sFlt-1 interaction (33). The present study found a positive correlation between serum ES and VEGF levels among patients with PCOS, indicating that the two molecules have an important synergistic relationship in regulating angiogenesis. Since abnormally elevated VEGF promotes angiogenesis, homeostatic regulation will prompt the 
production of ES (angiogenesis inhibitor) to counteract the accelerated event of angiogenesis. This would explain the significant increase in ES in PCOS patients (8). However, this compensatory effect does not restore the internal environment of the body to a normal physiological state (8). The dysregulation propagates abnormal vascularization and increased perfusion of the ovarian stroma (4).

The anti-angiogenic glycoprotein molecule TSP-1 is found in the extracellular matrix of both normal and tumor cells (34). The biological actions of TSP-1 arise from binding to its receptors and promoting apoptosis as well as cellular migration. TSP-1 has been reported to indirectly affect the biological activity of VEGF (34). The anti-angiogenic effects of TSP-1 have been confirmed in patients with endometriosis (35). Patients with chocolate cysts have been demonstrated to have high levels of TSP-1 and low levels of VEGF in their ovarian tissues (36). An imbalance in the expression of TSP-1 and VEGF may be a potential cause of endometriosis (35). Lower serum TSP-1 levels have been reported in PCOS patients compared to the normal population.

On the other hand, PCOS patients having insulin resistance have displayed a higher TSP-1 level compared to patients with insulin resistance who do not have PCOS $(36,37)$. Additionally, TSP-1 is known to be expressed in normal ovarian tissues, while TSP-1, together with its receptor CD36, plays an important regulatory role in ovarian function, including follicular growth, luteal formation, and ovarian angiogenesis (38). Based on the documented biological activities of TSP-1, it is thus speculated that the molecule plays an important role in the development of PCOS. The vascular phenomena observed in patients with PCOS may, therefore, be contributed to both TSP-1 and VEGF expression.

In our study, we noted that the LH/FSH ratio was significantly higher in all PCOS subtypes compared to healthy controls. This would help in the identification of PCOS patients from normal women. A recent case-control study (441 PCOS women and 422 non-PCOS women) was conducted to examine the diagnostic ability of the LH/FSH ratio in the identification of PCOS cases (39). An optimum LH/FSH ratio cut-off value of 1.33 was significant to diagnose PCOS (area under curve $=0.867$ ). Furthermore, a one-unit increase in LH/FSH ratio is associated with an increase in the risk of PCOS by 14.43 folds (95\% CI=9.3022.39). Despite the fact that this biomarker alone is not sufficient for a proper diagnosis of PCOS, the combination of various endocrine factors (such as $\mathrm{LH}$ and LH/FSH ratio) along with BMI, other anthropometric measures, and clinical features may provide an extra value in the establishment of a PCOS diagnosis.

The resolution of dysregulated angiogenesis, in general, as a potential therapeutic option has witnessed increasingly rapid advances in many pathological diseases, such as cancer $(40,41)$, inflammatory conditions, retinal diseases, and age-related macular degeneration (42-44). Since dysregulated ovarian angiogenesis is a main feature of PCOS, restoring normal angiogenesis has been suggested as a possible therapeutic approach for PCOS to improve ovulation and fertility in affected populations. In this context, a growing body of evidence has investigated the effect of various therapeutic approaches on ovarian angiogenesis, including laparoscopic ovarian drilling (45), metformin (10), VEGF inhibitor (46), platelet-derived growth factor BB (PDGFBB) (47), and oral contraceptive pills (9). In a recent case-control study, it was observed that both RI and PI were significantly increased in healthy women (control group), as well as in women who received oral contraceptive pills (30 mcg Ethinyl estradiol and drospirenone $3 \mathrm{mg}$ for 3 months) (9). This goes in line with our observation. In our study, all PCOS subtypes had significantly lower PI and RI at baseline (before treatment) compared to the control group. After a 3-month course of Diane-35 (Ethinyl estradiol), we noted that both PI and RI values increased in all groups compared to pre-treatment levels. These observations of low levels of Doppler measurements may reflect increased ovarian stromal vascularization in PCOS patients $(45,48-52)$. A few reports have studied the effects of OCP on ovarian blood flow in women with PCOS, and they have reported that OCP is correlated with reduced ovarian vasculature $(49,53)$. The reduction in PI and RI values at baseline in patients with PCOS could be explained by the main pathophysiologic mechanism of PCOS, as discussed above, with the involvement of various angiogenic markers in this process. Moreover, it has been proposed that higher levels of IR are of great importance in the regulation of ovarian vascularity in women with PCOS via the increase in androgen production and angiogenesis $(48,50,52)$. Another explanation for the reduced ovarian angiogenesis (increased PI and RI) could be the associated increase in the expression of the anti-angiogenic factor TSP-1, which increased significantly after Diane- 35 treatment, despite the lack of a significant correlation between both markers in our analysis, probably due to the small sample size. These observations suggest that the use of Diane- 35 treatment could be a potential therapeutic option for restoring normal angiogenesis in patients with PCOS.

Our study provides a helpful insight into the pathogenesis of PCOS by the variable expression of pro-angiogenic and antiangiogenic factors. We noted the ovarian blood flow indices (PI and RI), as well as TSP-1 levels, were reduced in PCOS patients compared to controls. On the other hand, ES levels were increased in all PCOS patients, while VEGF was only significantly increased in a subset of PCOS patients with hyperandrogenemia, as compared with healthy controls. That being said, our study had several limitations, the most important of which is the small number of included participants in each group, which was not sufficient enough to detect statically significant differences. Also, all lab values were monitored by a single clinician who was not blinded to the status of the patient. An important point to consider is that our results could be confounded by many un-analyzed variables, and therefore, more studies of larger sample sizes are still warranted to reach a definitive conclusion. Based on the aforementioned limitations, our findings should be interpreted with caution.

In conclusion, our study demonstrates that the dysregulation of ovarian angiogenesis is a central feature in the pathogenesis of PCOS, contributing to various phenotypes of the disease. Compared to healthy controls, low levels of Doppler measurements and changes in the expression level of various angiogenic and anti-angiogenic markers are also seen in women with PCOS. Diane-35 therapy resulted in an increase in Doppler measurements and TSP-1 biomarker levels in most PCOS phenotypes. However, variable observations regarding the 
expression levels of VEGF and ES were noted following a course of Diane-35 treatment. Finally, the imbalance of these biomarkers plays a role in the pathogenesis of PCOS, and the use of therapeutic options that affect ovarian angiogenesis would be of great importance in restoring normal follicular development, ovulation, and corpus luteum formation. More studies are still warranted to clarify the role of angiogenesis in different PCOS phenotypes and to develop new potential therapeutic strategies.

\section{DATA AVAILABILITY STATEMENT}

The original contributions presented in the study are included in the article/supplementary material. Further inquiries can be directed to the corresponding author.

\section{ETHICS STATEMENT}

The studies involving human participants were reviewed and approved by Second Affiliated Hospital of Harbin Medical

\section{REFERENCES}

1. Di Pietro M, Pascuali N, Parborell F, Abramovich D. Ovarian angiogenesis in polycystic ovary syndrome. Reproduction (2018) 155(5):R199-209. doi: 10.1530/REP-17-0597

2. Lin X, Zhang Y, Pan Y, He S, Dai Y, Zhu B, et al. Endometrial stem cellderived granulocyte-colony stimulating factor attenuates endometrial fibrosis via sonic hedgehog transcriptional activator Gli2. Biol Reprod (2018) 98 (4):480-90. doi: 10.1093/biolre/ioy005

3. Afiqah-Aleng N, Altaf-Ul-Amin M, Kanaya S, Mohamed-Hussein Z-A. Polycystic ovarian syndrome novel proteins and significant pathways identified using graph clustering approach. Reprod Bio Med Online (2020) 40(2):319-30 doi: 10.1016/j.rbmo.2019.11.012

4. Tal R, Seifer DB, Arici A. The emerging role of angiogenic factor dysregulation in the pathogenesis of polycystic ovarian syndrome. Seminars in reproductive medicine. New York, USA: Thieme Medical Publishers (2015). doi: 10.1055/s0035-1552582

5. Bruno JB, Matos MHT, Chaves RN, Celestino JJH, Saraiva MVA, Lima-Verde IB, et al. Angiogenic factors and ovarian follicle development. Anim Reprod (AR) (2018) 6(2):371-9.

6. Wang Y, Wang L, Chen C, Chu X. New insights into the regulatory role of microRNA in tumor angiogenesis and clinical implications. Mol Cancer (2018) 17(1):22. doi: 10.1186/s12943-018-0766-4

7. Sathyapalan T, Javed Z, Kilpatrick ES, Coady AM, Atkin SL. Endocannabinoid receptor blockade increases vascular endothelial growth factor and inflammatory markers in obese women with polycystic ovary syndrome. Clin Endocrinol (2017) 86(3):384-7. doi: 10.1111 /cen.13239

8. Chen F, Huang S, Chunrong H, Zheng S. Research of serum vascular endothelial growth factor and endostatin level and their effects on ovarian interstitial blood flow in patients with polycystic ovary syndrome. Clin Med China (2018) 34(2):170-2.

9. Okyay E, Gode F, Acet F, Bodur T, Cagliyan E, Sahan C, et al. The effect of drospirenone $(3 \mathrm{mg})$ with ethinyl estradiol $(30 \mathrm{mcg})$ containing pills on ovarian blood flows in women with polycystic ovary syndrome: a case controlled study. Eur J Obstet Gynecol Reprod Biol (2014) 180(3):93-9. doi: 10.1016/j.ejogrb.2014.06.027

10. Palomba S, Falbo A, La Sala GB. Effects of metformin in women with polycystic ovary syndrome treated with gonadotrophins for in vitro fertilisation and intracytoplasmic sperm injection cycles: a systematic review and meta-analysis of randomised controlled trials. Bjog (2013) 120(3):267-76. doi: 10.1111/1471-0528.12070
University. The patients/participants provided their written informed consent to participate in this study.

\section{AUTHOR CONTRIBUTIONS}

M-ML wrote and approved the manuscript. X-HC revised the manuscript. X-ML, F-FW, CW, YL, and SL collected data. P-LL, B-TD, Y-XW, and P-DG analyzed the patient data. All authors contributed to the article and approved the submitted version.

\section{FUNDING}

This study was supported by the Joint Guiding Project of Natural Science Foundation of Heilongjiang Province (no. LH2019H070) and the Research Fund for Young and Middle-Aged Innovative Science, the Second Affiliated Hospital of Harbin Medical University (no. KYCX 2018-03).

11. Azziz R. Diagnosis of polycystic ovarian syndrome: the Rotterdam criteria are premature. J Clin Endocrinol Metab (2006) 91(3):781-5. doi: 10.1210/jc.20052153

12. Cui LL, Chen ZJ. Polycystic ovary syndrome diagnostic criteria and diagnostic guidelines. J Int Reprod Health/Family Plann (2011) 30(05):405-8. doi: 10.3969/j.issn.1674-1889.2011.05.018

13. Macklon NS, Fauser BCJM. Medical approaches to ovarian stimulation for infertility. In: Yen and Jaffe"s reproductive endocrinology. Oxford: Elsevier Limited (2019). p. 689-724.e7. doi: 10.1016/b978-1-4160-4907-4.00028-0

14. Abdelnaby EA, El-Maaty AMA, Ragab RS, Seida AA. Dynamics of uterine and ovarian arteries flow velocity waveforms and their relation to follicular and luteal growth and blood flow vascularization during the estrous cycle in Friesian cows. Theriogenology (2018) 121:112-21. doi: 10.1016/ j.theriogenology.2018.08.003

15. Razik MA, Farag MAH, Sheta M. Uterine and ovarian arteries blood flow during the mid luteal phase in women with unexplained infertility. Middle East Fertility Soc J (2015) 20(3):209-12. doi: 10.1016/j.mefs.2014.05.004

16. Giampaolino P, Morra I, Della Corte L, Sparice S, Di Carlo C, Nappi C, et al. Serum anti-Mullerian hormone levels after ovarian drilling for the second-line treatment of polycystic ovary syndrome: a pilot-randomized study comparing laparoscopy and transvaginal hydrolaparoscopy. Gynecological Endocrinol (2017) 33(1):26-9. doi: 10.1080/09513590.2016.1188280

17. Garg N, Khaira HK, Kaur M, Sinha S. A Comparative Study on Quantitative Assessment of Blood Flow and Vascularization in Polycystic Ovary Syndrome Patients and Normal Women Using Three-Dimensional Power Doppler Ultrasonography. J Obstetrics Gynecology India (2018) 68(2):136-41. doi: 10.1007/s13224-017-1082-4

18. Katulski K, Czyzyk A, Podfigurna-Stopa A, Genazzani AR, Meczekalski B. Pregnancy complications in polycystic ovary syndrome patients. Gynecological Endocrinol (2015) 31(2):87-91. doi: 10.3109/09513590.2014. 974535

19. Dhingra D, Prateek S, Sinha R, Agarwal Y. Doppler flow velocities of uterine and ovarian arteries \& hormonal patterns in patients with Polycystic Ovary Syndrome (PCOS). Int J Healthcare Biomed Res (2017) 5(04):48-57. doi: 10.1016/ j.ijgo.2008.12.023

20. Bao L, Syed R, Aloahd MS. Analysis of VEGF gene polymorphisms and serum VEGF protein levels contribution in polycystic ovary syndrome of patients. Mol Biol Rep (2019) 46(6):5821-9. doi: 10.1007/s11033-019-05015-y

21. Holmes DI, Zachary I. The vascular endothelial growth factor (VEGF) family: angiogenic factors in health and disease. Genome Biol (2005) 6(2):209. doi: 10.1186/gb-2005-6-2-209 
22. Ferrara N. The role of VEGF in the regulation of physiological and pathological angiogenesis. Exs (2005) 94):209-31. doi: 10.1007/3-76437311-3_15

23. Greenaway J, Connor K, Pedersen HG, Coomber BL, LaMarre J, Petrik J. Vascular endothelial growth factor and its receptor, Flk-1/KDR, are cytoprotective in the extravascular compartment of the ovarian follicle. Endocrinology (2004) 145(6):2896-905. doi: 10.1210/en.2003-1620

24. Irani M, Seifer D, Grazi R, Irani S, Rosenwaks Z, Tal R. Vitamin D decreases serum VEGF correlating with clinical improvement in vitamin D-deficient women with PCOS: a randomized placebo-controlled trial. Nutrients (2017) 9 (4):334. doi: 10.3390/nu9040334

25. Mabjeesh NJ, Willard MT, Frederickson CE, Zhong H, Simons JW. Androgens stimulate hypoxia-inducible factor 1 activation via autocrine loop of tyrosine kinase receptor/phosphatidylinositol 3'-kinase/protein kinase B in prostate cancer cells. Clin Cancer Res (2003) 9(7):2416-25.

26. Shafighi M, Olariu R, Brun C, Fathi AR, Djafarzadeh S, Jakob SM, et al. The role of androgens on hypoxia-inducible factor (HIF)- $1 \alpha$-induced angiogenesis and on the survival of ischemically challenged skin flaps in a rat model. Microsurgery (2012) 32(6):475-81. doi: 10.1002/micr.21996

27. Ferrara N. Vascular endothelial growth factor: basic science and clinical progress. Endocr Rev (2004) 25(4):581-611. doi: 10.1210/er.2003-0027

28. Mitani T, Yamaji R, Higashimura Y, Harada N, Nakano Y, Inui H. Hypoxia enhances transcriptional activity of androgen receptor through hypoxiainducible factor- $1 \alpha$ in a low androgen environment. J Steroid Biochem Mol Biol (2011) 123(1-2):58-64. doi: 10.1016/j.jsbmb.2010.10.009

29. Park C, Kim Y, Shim M, Lee Y. Hypoxia enhances ligand-occupied androgen receptor activity. Biochem Biophys Res Commun (2012) 418(2):319-23. doi: 10.1016/j.bbrc.2012.01.019

30. Pan F, Yang W, Li W, Yang X-Y, Liu S, Li X, et al. Conjugation of gold nanoparticles and recombinant human endostatin modulates vascular normalization via interruption of anterior gradient 2-mediated angiogenesis. Tumor Biol (2017) 39(7):1-9. doi: 10.1177/101042831770-8547

31. Myren M, Kirby DJ, Noonan ML, Maeda A, Owens RT, Ricard-Blum S, et al. Biglycan potentially regulates angiogenesis during fracture repair by altering expression and function of endostatin. Matrix Biol (2016) 52:141-50. doi: 10.1016/j.matbio.2016.03.008

32. Ha JM, Jin SY, Lee HS, Shin HK, Lee DH, Song SH, et al. Regulation of retinal angiogenesis by endothelial nitric oxide synthase signaling pathway. Korean J Physiol Pharmacol (2016) 20(5):533-8. doi: 10.4196/kjpp.2016. 20.5.533

33. Walia A, Yang JF, Huang Y-h, Rosenblatt MI, Chang J-H, Azar DT. Endostatin's emerging roles in angiogenesis, lymphangiogenesis, disease, and clinical applications. Biochim Biophys Acta (BBA)-General Subj (2015) 1850(12):2422-38. doi: 10.1016/j.bbagen.2015.09.007

34. Choi SH, Tamura K, Khajuria RK, Bhere D, Nesterenko I, Lawler J, et al. Antiangiogenic variant of TSP-1 targets tumor cells in glioblastomas. Mol Ther (2015) 23(2):235-43. doi: 10.1038/mt.2014.214

35. Marí-Alexandre J, García-Oms J, Barceló-Molina M, Gilabert-Aguilar J, Estellés A, Braza-Boíls A, et al. MicroRNAs and angiogenesis in endometriosis. Thromb Res (2015) 135:S38-40. doi: 10.1016/S0049-3848(15) 50439-8

36. Tahergorabi Z, Salmani F, Jonaidabad SH, Behdani B, Yazdi P, Zardast M, et al. Association of serum levels of vascular endothelial growth factor and thrombospondin-1 to body mass index in polycystic ovary syndrome: a casecontrol study. Obstetrics Gynecol Sci (2019) 62(6):420-8. doi: 10.5468/ ogs.2019.62.6.420

37. Liu M, Gao J, Zhang Y, Li P, Wang H, Ren X, et al. Serum levels of TSP-1, NF$\kappa B$ and TGF- $\beta 1$ in polycystic ovarian syndrome (PCOS) patients in northern China suggest PCOS is associated with chronic inflammation. Clin Endocrinol (2015) 83(6):913-22. doi: 10.1111/cen.12951

38. Rodler D, Sinowatz F. Localization of thrombospondin-1 and its receptor CD36 in the ovary of the ostrich (Struthio camelus). Anatomia histologia Embryologia (2018) 47(2):124-32. doi: 10.1111/ahe.12329

39. Le MT, Le VNS, Le DD, Nguyen VQH, Chen C, Cao NT. Exploration of the role of anti-Mullerian hormone and LH/FSH ratio in diagnosis of polycystic ovary syndrome. Clin Endocrinol (Oxf) (2019) 90(4):579-85. doi: 10.1111/ cen.13934
40. Albiges L, Salem M, Rini B, Escudier B. Vascular endothelial growth factortargeted therapies in advanced renal cell carcinoma. Hematol Oncol Clin North Am (2011) 25(4):813-33. doi: 10.1016/j.hoc.2011.04.006

41. Socinski MA. Multitargeted receptor tyrosine kinase inhibition: an antiangiogenic strategy in non-small cell lung cancer. Cancer Treat Rev (2011) 37(8):611-7. doi: 10.1016/j.ctrv.2011.04.003

42. Bauditz J, Lochs H. Angiogenesis and vascular malformations: antiangiogenic drugs for treatment of gastrointestinal bleeding. World J Gastroenterol (2007) 13(45):5979-84. doi: 10.3748/wjg.v13.45.5979

43. Mitchell P. A systematic review of the efficacy and safety outcomes of antiVEGF agents used for treating neovascular age-related macular degeneration: comparison of ranibizumab and bevacizumab. Curr Med Res Opin (2011) 27 (7):1465-75. doi: 10.1185/03007995.2011.585394

44. Ribeiro JA, Messias A, Jorge R. Antiangiogenic drugs and advanced proliferative diabetic retinopathy. Arq Bras Oftalmol (2011) 74(2):143-6. doi: 10.1590/S0004-27492011000200017

45. El Behery MM, Diab AE, Mowafy H, Ebrahiem MA, Shehata AE. Effect of laparoscopic ovarian drilling on vascular endothelial growth factor and ovarian stromal blood flow using 3-dimensional power Doppler. Int $J$ Gynaecol Obstet (2011) 112(2):119-21. doi: 10.1016/j.ijgo.2010.08.018

46. Abramovich D, Rodriguez Celin A, Hernandez F, Tesone M, Parborell F. Spatiotemporal analysis of the protein expression of angiogenic factors and their related receptors during folliculogenesis in rats with and without hormonal treatment. Reproduction (2009) 137(2):309-20. doi: 10.1530/REP08-0130

47. Di Pietro M, Scotti L, Irusta G, Tesone M, Parborell F, Abramovich D. Local administration of platelet-derived growth factor B (PDGFB) improves follicular development and ovarian angiogenesis in a rat model of Polycystic Ovary Syndrome. Mol Cell Endocrinol (2016) 433:47-55. doi: 10.1016/j.mce.2016.05.022

48. Adali E, Kolusari A, Adali F, Yildizhan R, Kurdoglu M, Sahin HG. Doppler analysis of uterine perfusion and ovarian stromal blood flow in polycystic ovary syndrome. Int J Gynaecol Obstet (2009) 105(2):154-7. doi: 10.1016/ j.ijgo.2008.12.023

49. Battaglia C, Mancini F, Fabbri R, Persico N, Busacchi P, Facchinetti F, et al. Polycystic ovary syndrome and cardiovascular risk in young patients treated with drospirenone-ethinylestradiol or contraceptive vaginal ring. A prospective, randomized, pilot study. Fertil Steril (2010) 94(4):1417-25. doi: 10.1016/j.fertnstert.2009.05.044

50. Loverro G, Vicino M, Lorusso F, Vimercati A, Greco P, Selvaggi L. Polycystic ovary syndrome: relationship between insulin sensitivity, sex hormone levels and ovarian stromal blood flow. Gynecol Endocrinol (2001) 15(2):142-9. doi: $10.1080 / 713602806$

51. Ozcimen EE, Uckuyu A, Ciftci FC, Zeyneloglu HB. The effect of metformin treatment on ovarian stromal blood flow in women with polycystic ovary syndrome. Arch Gynecol Obstet (2009) 280(2):263-9. doi: 10.1007/s00404008-0904-0

52. Parsanezhad ME, Bagheri MH, Alborzi S, Schmidt EH. Ovarian stromal blood flow changes after laparoscopic ovarian cauterization in women with polycystic ovary syndrome. Hum Reprod (2003) 18(7):1432-7. doi: 10.1093/ humrep/deg244

53. Battaglia C, Genazzani AD, Artini PG, Salvatori M, Giulini S, Volpe A. Ultrasonographic and color Doppler analysis in the treatment of polycystic ovary syndrome. Ultrasound Obstet Gynecol (1998) 12(3):180-7. doi: 10.1046/ j.1469-0705.1998.12030180.x

Conflict of Interest: The authors declare that the research was conducted in the absence of any commercial or financial relationships that could be construed as a potential conflict of interest.

Copyright $\odot 2021$ Liu, Chen, Lu, Wang, Wang, Liu, Li, Du, Liang, Gong and Wang. This is an open-access article distributed under the terms of the Creative Commons Attribution License (CC BY). The use, distribution or reproduction in other forums is permitted, provided the original author(s) and the copyright owner(s) are credited and that the original publication in this journal is cited, in accordance with accepted academic practice. No use, distribution or reproduction is permitted which does not comply with these terms. 